\title{
CAN WE DEFINE AN INERTIAL REFERENCE SYSTEM?
}

\author{
LI LINGHUAI , TONG FU \\ Purple Mountain Observatory \\ Academia Sinica \\ Nanjing, China
}

The topic (Seidelmann, 1986) is one of the unresolved questions in the field of celestial mechanics because the definitions of an inertial system are all controversial (Mach, 1893; Eichhorn, 1984). Eichhorn's definition enlarges its connotation and is not acceptable either. Connotation of a concept is endowed through defining it. One of the reasons why the existing definitions of an inertial system are all controversial is that the connotations that are endowed by them are not unique, in other words, they do not draw up the same area of ideas. Taking the interplay of all of them for its connotation is an acceptable solution.

According to general relativity, as far as a reference system is concerned, we always divide the Universe into two parts: the background and the test bodies we are interested in. According to the metric theories of gravity, the action of matter in the Universe to the test particles is not direct but through thespace-time which is determined by it. Thus reference systems are space-time in nature and should be classified in terms of its intrinsic nature, the scalar curvature. As a result, an inertial system can be defined as: Flat background space-time is called an inertial reference system.

In order to make the definition not so abstract in appearence, let's associate it with something familiar to us through discussing its coordinate representation. To specify a reference system is to give a scalar curvature field $R(p)$, for example, $R(p)$ equals zero for the inertial system according to the definition above. According to Riemannian geometry, the curvature tensor $R_{1 \mid}$ can be constructed through making use of $\mathrm{R}$ and $g_{i j}: \mathrm{R}_{\mathrm{ij}}=g_{i j} R / 4$.

On the other hand, $R_{i j}$ is defined as functions of the metric and its first and second derivatives, $\mathrm{R}_{\mathrm{ij}}=\mathrm{R}_{\mathrm{ij}}\left(g_{k l}, \mathrm{D} g_{k f}, \mathrm{D}^{2} g_{k l}\right)$. Thus we have following equations: $\mathrm{R}_{\mathrm{ij}}\left(g_{k l}, \mathrm{D} g_{k l}, \mathrm{D}^{2} g_{k l}\right)=g_{i j} R / 4$, which are independent of any coordinate system. In order to specify a coordinate system, a coordinate condition consisting of four additional equations must be given. Consequently, it can be inferred that different coordinate conditions in GTR are related to different measurement conventions and the coordinate transformations are related to transformations between measurement conventions.

\section{References}

Seidelmann, P.K.: 1986, Celes. Mech.,39, 141.

Mach, E.: 1893, The Science of Mechanics , trans. by T.J. McCormack (2nd ed., Open Court Publishing Co.).

Eichhorn, H.: 1984, Celes. Mech., 34, 11. 